\title{
A numerical model to investigate the effect of honing angle on the hydrodynamic lubrication between a combustion engine piston ring and cylinder liner
}

\author{
Andrew Spencer ${ }^{1}$, Andreas Almqvist ${ }^{1}$, Roland Larsson ${ }^{1}$ \\ ${ }^{1}$ Luleå University of Technology, 97187 Luleå, Sweden \\ Corresponding author: andrew.spencer@ltu.se
}

A numerical model has been developed to investigate the effect of cylinder liner honing angle on hydrodynamic lubrication between piston ring and cylinder liner. The Reynolds equation was solved in 2D with periodic boundary conditions. An artificial surface texture was generated, based on a real surface measured with white light interferometry. Cavitation was modelled with the Vijayaraghavan and Keith algorithm. Honing angles between $25-75^{\circ}$ were investigated to find the effect of honing angle on film thickness.

Keywords: Cylinder Liner, Honing, Texture

\section{NOTATION AND UNITS}

$\mathrm{d}$ - Bore diameter (m)

$\mathrm{F}_{\mathrm{t}}-$ Ring Tension $(\mathrm{N})$

$\mathrm{G}-$ Switch function

$\mathrm{h}-$ Film thickness (m)

$1-$ Ring width (m)

$\mathrm{P}_{\mathrm{IN}}-$ Inlet Pressure $(\mathrm{Pa})$

$\mathrm{P}_{\text {Out }}$ - Outlet Pressure (Pa)

$\mathrm{t}$ - Time (s)

$\mathrm{U}-$ Entraining velocity $(\mathrm{m} / \mathrm{s})$

$\alpha-$ Honing angle (deg)

$\beta$ - Compressibility factor $(\mathrm{Pa})$

$\rho_{\mathrm{c}}-$ Cavitation density $\left(\mathrm{kg} / \mathrm{m}^{3}\right)$

$\mu$ - Dynamic Viscosity $(\mathrm{Pa} \cdot \mathrm{s})$

$\theta$ - Film fraction

\author{
$\underline{\text { Subscripts }}$ \\ * - Previous time step \\ $\mathrm{e}$ - one node downstream \\ w - one node upstream \\ $\mathrm{n}$ - one node to the left \\ $s$ - one node to the right \\ prev - previous iteration
}

\section{INTRODUCTION}

Power losses from an internal combustion engine can account for a large percentage of the total power output of the engine, varying from $10 \%$ at full load to $100 \%$ at idle [1]. Frictional losses account for a large percentage of these and the piston ring to cylinder liner (PRCL) conjunction is the single largest contributor to frictional losses in an IC engine, accounting for $20-40 \%$ of the total 
frictional losses [2]. The compression rings are responsible for $4-5 \%$ of all losses in a multicylinder engine [3]. If parasitic losses can be reduced by $4 \%$ then a fuel efficiency increase of $1 \%$ can be realised [3]. Therefore studying this tribological contact has great potential for reducing friction and improving fuel economy in an engine, something that is at the forefront of engine design today.

Typically a machining process known as honing is used to apply the desired finish to the cylinder liner surface. The grooves that the honing process leaves behind are crucial in controlling the amount of oil available in the contact, by both retaining oil on the liner surface and improving the distribution of oil. Another function of the honing texture is to allow wear debris, generated during boundary lubrication around TDC, to be channelled away from the conjunction so as to cause only minimal damage and scratching to the smooth plateaux which are crucial for fluid film generation. In this study the effect of applying the honing to hydrodynamic lubrication is investigated.

Very few numerical studies have investigated the effect of honing parameters, and in particular honing angle, on lubrication performance. Therefore this may be an area that has great potential for optimisation, and changing a parameter such as honing angle should not add any significant costs to cylinder liner manufacture. Most existing PRCL models do not consider the non-Gaussian roughness patterns typical of a honed surface.

Michail and Barber [4] undertook a study with similar objectives, implementing an analytical emulation of a honed surface. Their study used the Patir and Cheng [5] Average Flow model and took the honed surface to be a combination of cosine waves with the peaks removed to simulate the plateaux. It is suggested that the artificial surface texture presented here could be a more realistic representation of a honed surface than their approach.

\section{THEORY}

\subsection{Surface Texture}

An artificial surface texture was generated based on real cylinder liner surface topography. The surface texture of a cylinder liner was sampled using white light interferometry with a Wyko NT1100 to investigate the typical honing pattern, illustrated in Figure 1.

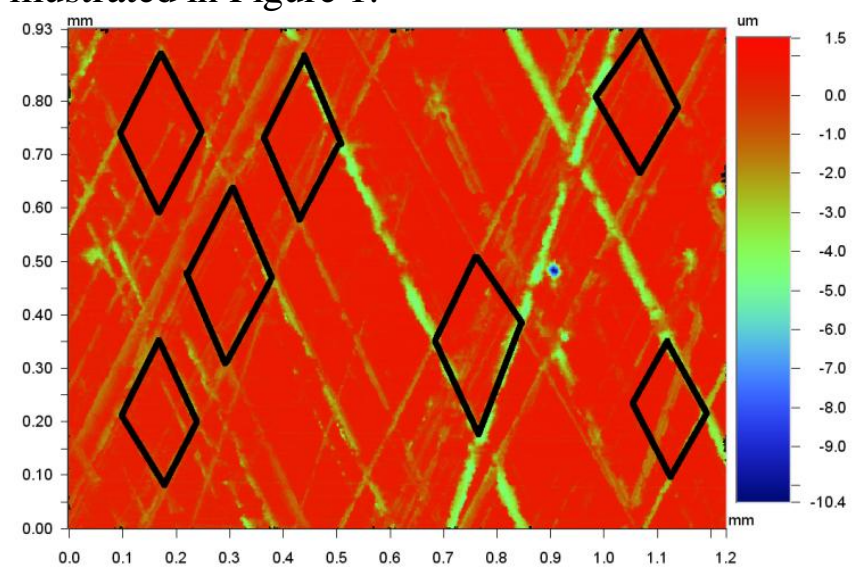

Figure 1 - White light interferometry image of cylinder liner surface

Although the diamond patterns generated on the surface by the honing operation are not uniform, a typical diamond, as highlighted in the image, has an area of $0.048 \mathrm{~mm}^{2}$. In this study, to keep textures with different honing angles comparable, the area of each diamond will be kept constant at this value. To generate a texture 'groove' in the surface, the following equation is used [6]:

$$
h_{T}(x, y)=10^{-w(x+k y)^{2}} \cos [2 \pi(x+k y)] .
$$

Two variables require some explanation. The $w$ coefficient is used to adjust the width of the honing groove. This is done iteratively to achieve the desired percentage of plateau. The parameter $\mathrm{k}$ is a function of the angle of the honing groove, and is derived as:

$$
k=\frac{1}{\tan \alpha}
$$

Equation (1) is used to create one diagonal groove, illustrated in Figure 2. 


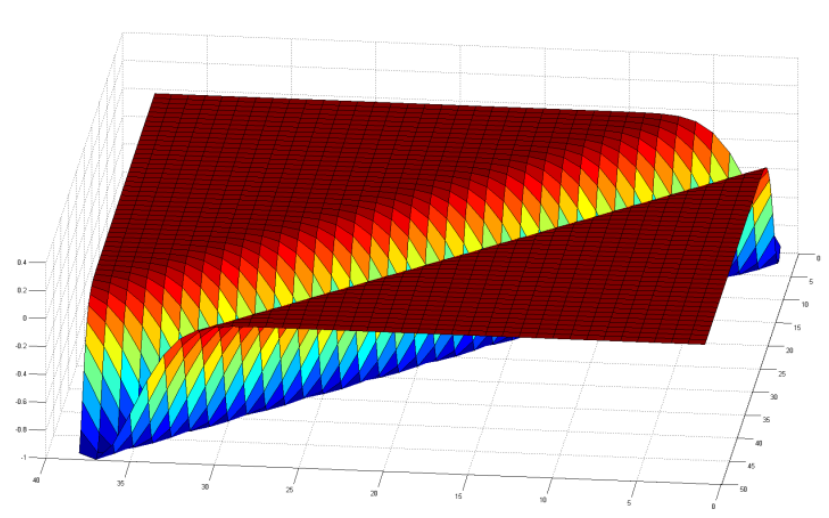

Figure 2 - Artificial honing groove

To create the diamond shape as seen on the real surface this single groove is mirrored in the $\mathrm{x}$ direction and then $\mathrm{y}$ direction. This single diamond is tiled to create a complete surface for the ring to slide over (Figure 3). The surface is long enough for the ring to be able to slide far enough, through enough time steps, for a steady state periodic solution to be reached. The surface is only one diamond wide in the circumferential direction; periodic boundary conditions are implemented in the solution so this is all that is required.

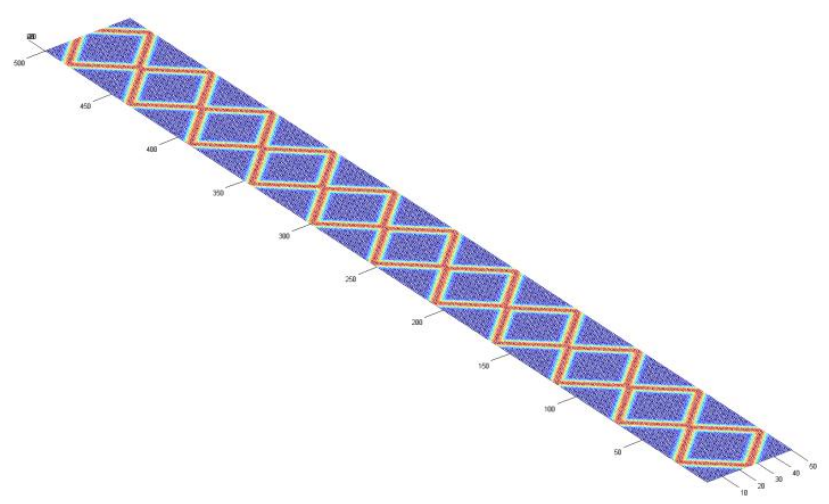

Figure 3 - Generated Surface Texture

Information regarding the depth of the honing scratches can also be obtained from the Wyko measurements; Figure 4 shows a typical crosssection.

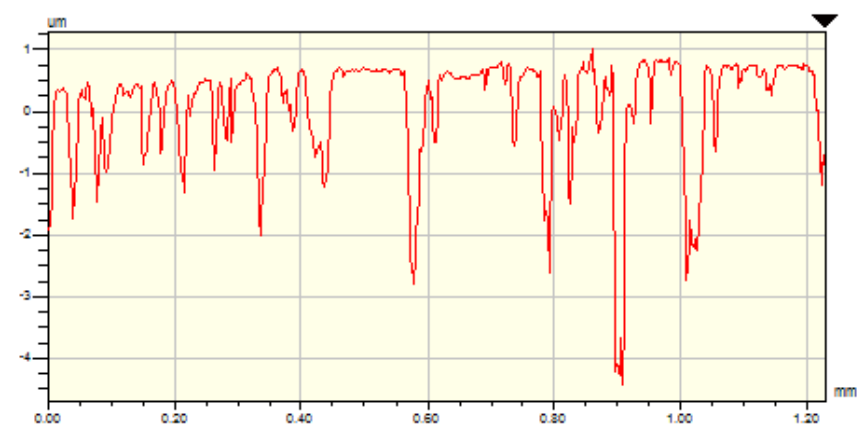

Figure 4 - Cross-section of measured liner surface

It can be observed that the honing depth has some variation. In this simulation a value of $2 \mu \mathrm{m}$ has been used as a representative depth.

\subsection{Cavitation Algorithm}

The Vijayaraghavan and Keith [7] cavitation algorithm was used to solve the Reynolds equation, implementing cavitation boundary conditions at film rupture and reformation. The approach is similar to the Elrod algorithm [8] but with two advantages; compressibility is considered in the full film zone and the discretisation is rigorously derived, rather than being the result of considerable experimentation. The Reynolds equation is written as;

$$
\begin{array}{r}
\frac{d \rho_{c} h \theta}{d t}+\frac{d}{d x}\left(\frac{\rho_{c} h U}{2} \theta-\frac{\rho_{c} \beta h^{3} g}{12 \mu} \frac{d \theta}{d x}\right)+ \\
\frac{d}{d y}\left(-\frac{\rho_{c} \beta h^{3} g}{12 \mu} \frac{d \theta}{d y}\right)=0
\end{array}
$$

where $g$ is a switch function becoming 1 in the full-film zone and 0 in the cavitated zone. The type of zone is calculated from the value of $\theta$, above 1 the region is full-film and below 1 the region is cavitated. The film pressure is found from $\theta$;

$$
P=P_{c}+g \beta \ln \theta
$$

Once the discretization has been applied Eq. (3) can be written in the form;

$$
\begin{gathered}
a_{w} \theta_{i-1, j}+a_{e} \theta_{i+1, j}+a_{n} \theta_{i, j-1}+a_{s} \theta_{i, j+1}+ \\
a_{p} \theta_{i, j}=r h s,
\end{gathered}
$$


where;

$$
\begin{gathered}
a_{w}=U \cdot\left[c \cdot h_{i-1, j}\right] / 4 \Delta x-C 1\left[h_{w} g_{w}\right] \\
a_{e}=U \cdot\left[a \cdot h_{i+1, j}\right] / 4 \Delta x-C 1\left[h_{e} g_{e}\right] \\
a_{n}=-C 2\left[h_{n} g_{n}\right] \\
a_{s}=-C 2\left[h_{s} g_{s}\right] \\
a_{p}=U \cdot \frac{\left[b \cdot h_{i, j}\right]}{4 \Delta x}+C 1\left[\left(h_{e}+h_{w}\right) g_{p}\right] \\
+C 2\left[\left(h_{n}+h_{s}\right) g_{p}\right]-h_{p} / \Delta t \\
r h s=-C 1\left[h_{e} g_{e}-\left(h_{e}+h_{w}\right) g_{p}+h_{w} g_{w}\right] \\
-C 1\left[h_{s} g_{s}-\left(h_{n}+h_{s}\right) g_{p}\right. \\
\left.+h_{n} g_{n}\right]-\left(h_{p}^{*} \cdot \theta_{i, j}^{*}\right) / \Delta t
\end{gathered}
$$

Couette flow coefficients:

$$
\begin{gathered}
a=\left(g_{i+1, j}+g_{i, j}\right) / 2 \\
b=2-\left(g_{i+1, j}+2 g_{i, j}+g_{i-1, j}\right) / 2 \\
c=-\left[2-\left(g_{i, j}+g_{i-1, j}\right) / 2\right]
\end{gathered}
$$

Pouiselle flow coefficients:

$$
\begin{aligned}
& C 1=\frac{\beta}{12 \mu \Delta x^{2}} \\
& C 2=\frac{\beta}{12 \mu \Delta y^{2}}
\end{aligned}
$$

\section{$\underline{\text { Boundary conditions }}$}

A pressure is specified at the inlet and outlet points based on typical gas pressures encountered (see Figure 8). Periodic boundary conditions are implemented in the circumferential (perpendicular to entraining motion) direction. The inlet is assumed to be in the fully flooded condition.

\section{Film thickness}

The ring is assumed to have a parabolic shape. The total film thickness is a combination of this shape, the contribution from the surface texture and a minimum film thickness, given by equation (6).

$$
h=h_{\min }+\mathrm{ax}^{2}+h_{T} .
$$

An iterative procedure is used to solve the system of equations. The problem is solved over a grid of $1000 \times 50$ nodes. Initially $g$ is assumed to equal 1 everywhere. The values of $\theta$ are then calculated at all grid points using Gaussian elimination and $\mathrm{g}$ is updated based on these new values. This process is repeated until convergence is reached, which is assumed to be when the following becomes true:

$$
\frac{\iint P d x d y-\iint P_{\text {prev }} d x d y}{\iint P_{\text {prev }} d x d y}<10^{-5}
$$

\subsection{Force Balance and Time dependence}

An iterative procedure is used to calculate the value of $h_{\text {min }}$ required for the lubricant film pressure to support the opposing load, a combination of the gas pressure acting on the back of the ring and ring tension. This balance is defined as:

$$
\iint \mathrm{Pdxdy}=P_{\text {in }}+\frac{F_{t}}{(d / 2) l}
$$

The fzero algorithm in Matlab is used to find the required $h_{\min }$ to balance the forces. This is a combination of the bisection, secant, and inverse quadratic interpolation methods. Once the force balance has been satisfied the solution increments one time step. A new surface texture is calculated as the ring has slid over a proportion of the texture pattern and the force balance is then solved again. The time is incremented until the ring has slid over five full texture diamonds, enough for a periodic value of $h_{\min }$ to become apparent. Figure 8 in the Appendix lists all of the parameters used in the solution. The simulation is run with parameters that represent typical conditions at the mid-stroke of the engine cycle. The speed is kept constant and thus the result is at a stationary condition. The repeating pattern is, however, necessary so that a time dependent solution can be solved. The conditions used were chosen as the model presented is currently only capable of 
simulating hydrodynamic lubrication; the mixed and boundary lubrication regimes at Top Dead Centre (TDC) and Bottom Dead Centre (BDC) were intentionally avoided.

\section{RESULTS}

The honing angle of the cylinder liner measured with white light intereferometry (Figure 1) was $50^{\circ}$. Honing angles of $\pm 25^{\circ}$ of this existing case have been investigated, in increments of $5^{\circ}$. A typical pressure distribution is illustrated in Figure 5.

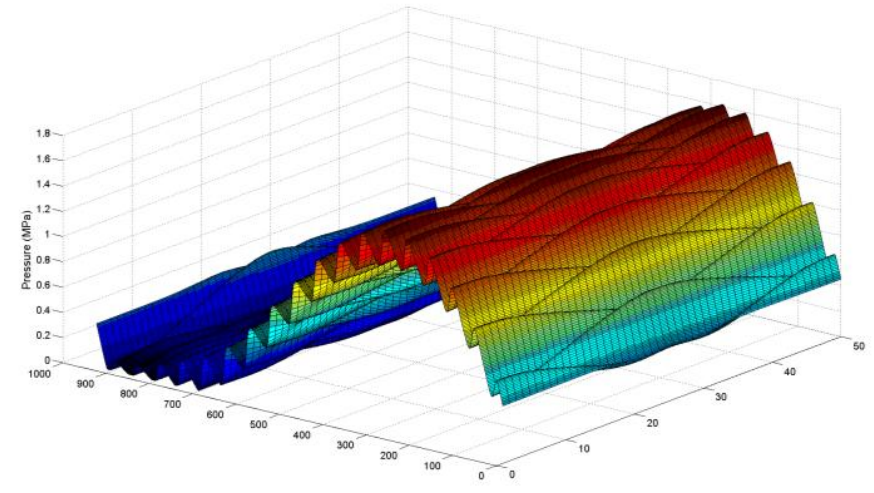

Figure 5 - Calculated lubricant pressure across ring width

The simulation shows that a steady state solution is obtained after the ring has transversed approximately two complete texture patterns, or 20 time steps. Figure 6 shows the minimum film thickness, $h_{\text {min }}$, as a function of time.

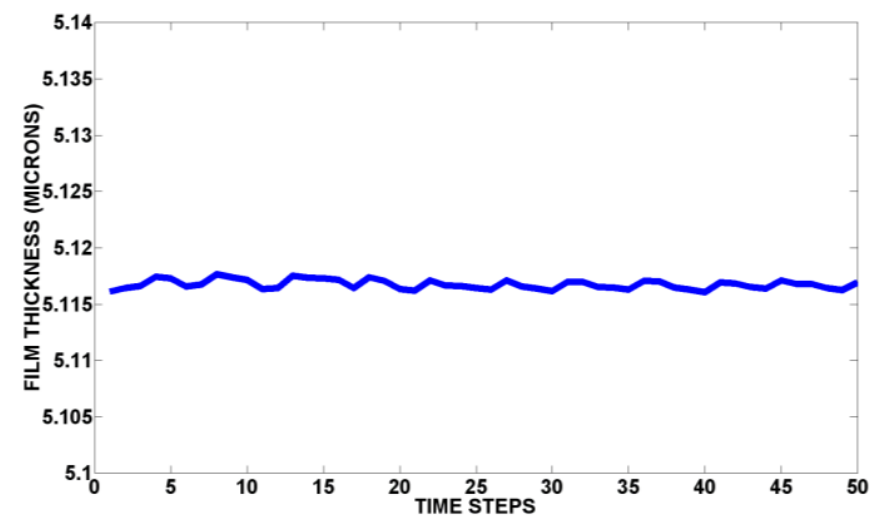

Figure 6 - Minimum Film Thickness as a function of time steps
From Figure 6 it can be seen that a fairly steady state of film thickness occurs after 20 time steps, with oscillations occurring periodically as the ring passes over each honing diamond. This pattern was observed for all of the honing angles investigated. Therefore in Figure 7 the average minimum film thickness is calculated for each honing angle from the 20th time step onwards; so that only the steady state points are considered.

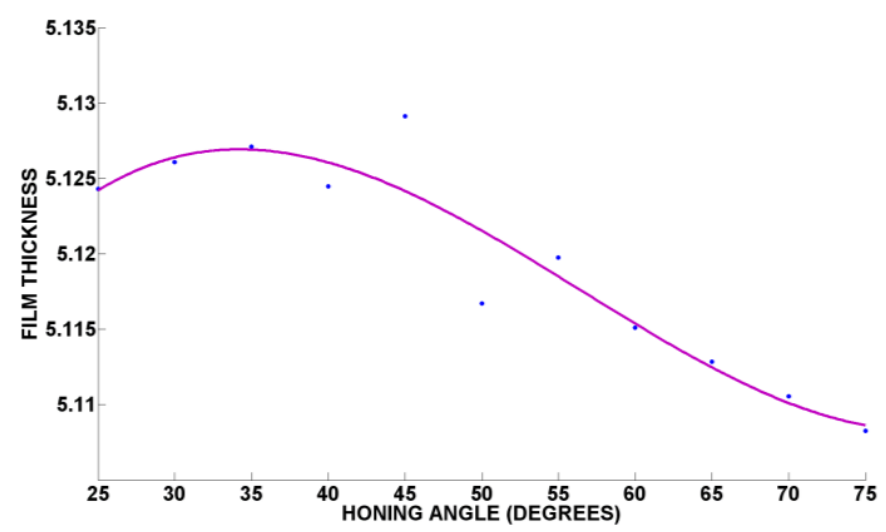

Figure 7 - Average Film Thickness verses Honing Angle

\section{DISCUSSION}

The first issue to highlight is that the variation in film thickness with honing angle is minimal. A 20 $\mathrm{nm}$ variation was observed across the range of honing angles $-0.4 \%$ of the total minimum film thickness of $\approx 5 \mu \mathrm{m}$. A curve has been fitted to the calculated average results however the values for $45^{\circ}$ and $50^{\circ}$ deviate significantly from this. However the results generally show that the film thickness is greater with a smaller honing angle. This is supported by the conclusions in [4] which also show that a smaller angle leads to a greater oil film thickness.

\section{CONCLUSION}

It has been shown that the effect of the honing angle in the middle of the piston stroke is negligible compared to the large hydrodynamic film already developed. A simulation run closer to TDC or BDC, thereby running at conditions closer to mixed lubrication, might yield a greater effect 
from the honing angle parameter as the surface grooves become a bigger percentage of the total film thickness. It would be also be of value to investigate angles far away from $50^{\circ}$, such as $140^{\circ}$, which can be machined as a product of helical slide honing. Such an angle has been shown to reduce oil consumption during bench tests [9].

\section{ACKNOWLEDGEMENTS}

The authors would like to thank Stiftelsen för Strategisk Forskning (SSF) and ProViking for funding this work and Scania AB.

\section{APPENDIX}

\begin{tabular}{|l|l|}
\hline \multicolumn{1}{|c|}{ Parameter } & \multicolumn{1}{c|}{ Value } \\
\hline Plateau depth & $2 \mu \mathrm{m}$ \\
\hline Area of one diamond texture & $0.048 \mathrm{~mm}^{2}$ \\
\hline Compressibility Factor $(\beta)$ & $6.9 \times 10^{7} \mathrm{~Pa}$ \\
\hline Dynamic Viscosity $(\mu)$ & $0.04 \mathrm{~Pa} \cdot \mathrm{s}$ \\
\hline Inlet Pressure $\left(\mathrm{P}_{\mathrm{IN}}\right)$ & $437.5 \mathrm{kPa}$ \\
\hline Outlet Pressure $\left(\mathrm{P}_{\mathrm{OUT}}\right)$ & $357.0 \mathrm{kPa}$ \\
\hline Simulation time & $1.5 \times 10^{-4} \mathrm{~s}$ \\
\hline No. of time steps & 50 \\
\hline Ring width & $4 \mathrm{~mm}$ \\
\hline Ring effective width & $2.7 \mathrm{~mm}$ \\
\hline Piston speed $(\mathrm{U})$ & $5 \mathrm{~m} / \mathrm{s}$ \\
\hline Tangential Ring Force $\left(\mathrm{F}_{\mathrm{t}}\right)$ & $10 \mathrm{~N}$ \\
\hline Bore diameter $(\mathrm{d})$ & $0.05 \mathrm{~m}$ \\
\hline
\end{tabular}

Figure 8 - Solution Parameters

\section{REFERENCES}

1. Heywood, J.B. Internal Combustion Engine Fundamentals. s.1. : McGraw-Hill, 1988.

2. Bolander, N.W., Steenwyk, B.D., Sadeghi, F. and Gerber, G.R. Lubrication regime transistions at the piston ring / cylinder liner interface. Proc. IMechE Part J: Engineering Tribology. 219, 2005. 3. Mishra, P.C., Rahnejat, H. and King, P.D. Tribology of the ring/bore conjunction subject to a mixed regime of lubrication. Proc. IMechE. Part C: Journal of Mechanical Engineering Science. 223, 2009.

4. Michail, S.K. and Barber, G.C. The Effects of Roughness on Piston Ring Lubrication - Part II: The Relationship between Cylinder Wall Surface Topography and Oil Film Thickness. Tribology Transactions. 1995, Vol. 38.

5. Patir, N. and Cheng, H.S. An Average Flow Model for Determining Effects of ThreeDimensional Roughness on Partial Hydrodynamic Lubrication. Transactions of the ASME. 100, 1978.

6. Almqvist, A., Essel, E.K., Fabricius, J. and Wall, P. Reiterated homogenization applied in hydrodynamic lubrication. Proc. IMechE Part J: Engineering Tribology. 222, 2008.

7. Vijayaraghavan, D. and Keith, T.G. Development and Evaluation of a Cavitation Algorithm. Tribology Transactions. 32, 1989.

8. Elrod, H.G. A Cavitation Algorithm. Transactions of the ASME. 103, 1981.

9. Haasis, G. and Weigmann, U.P. New honing technique reduces oil consumption. Industrial Diamond Review. 3, 99. 